\title{
Effect of Severe Renal Impairment on the Safety, Tolerability, and Pharmacokinetics of AMG 986
}

\author{
Ashit Trivedi $^{1} \cdot$ Omar Mather $^{1} \cdot$ Silvia Vega ${ }^{1} \cdot$ Mary Ann Simiens $^{1} \cdot$ Jennifer Hellawell $^{2} \cdot$ Edward Lee $^{1}$
}

Accepted: 21 December 2021 / Published online: 29 January 2022

(c) The Author(s) 2022

\begin{abstract} remains unknown. $\mathrm{mL} / \mathrm{min} / 1.73 \mathrm{~m}^{2}$ ). all adverse events were mild in severity. the need for dose adjustments.

\section{Key Points}

AMG $986200 \mathrm{mg}$ exposure was similar in participants with severe renal impairment and normal renal function.

The results of this study support the enrollment of heart failure patients with renal impairment to clinical trials of AMG 986 without the need for dose adjustments.
\end{abstract}

Introduction AMG 986 is a first-in-class, novel apelin receptor small molecule agonist initially developed for the treatment of heart failure (HF). The safety and pharmacokinetics (PK) of AMG 986 in participants with renal impairment (RI)

Methods This phase 1 study compared the safety and PK of AMG $986200 \mathrm{mg}$ in six participants with severe RI (estimated glomerular filtration rate [eGFR] $15-29 \mathrm{~mL} / \mathrm{min} / 1.73 \mathrm{~m}^{2}$ ) versus six participants with normal renal function (eGFR $\geq 90$

Results Following a single oral dose of AMG $986200 \mathrm{mg}$ on day 1, the maximum observed concentration increased 1.41fold (90\% confidence interval [CI] 0.88-2.27) and the area under the curve from time zero to infinity increased 1.23 -fold (90\% CI 0.73-2.06) in participants with severe RI versus normal renal function. AMG 986 had an acceptable safety profile;

Conclusions The results of this study support the enrollment of HF patients with RI to clinical trials of AMG 986 without

Trial Registration Number NCT03318809 (registered: October 24, 2017).

\footnotetext{
Ashit Trivedi

ashitt@amgen.com

1 Amgen Inc, Thousand Oaks, CA, USA

2 Amgen Inc, San Francisco, CA, USA
}

\section{Introduction}

Chronic heart failure (HF) is a complex syndrome that results in inadequate systemic blood flow when neurohormonal mechanisms are no longer able to deliver an adequate physiological response [1]. The prognosis for patients with HF remains poor and there is an unmet need for novel therapies [2]. The apelin receptor is a G-protein coupled receptor that counteracts the pressor effect of angiotensin II, attenuates ischemic injury, and contributes to neovascularization in HF [3, 4]. AMG 986 is a first-in-class, novel apelin receptor small molecule agonist that binds and activates the apelin receptor to improve cardiac function by increasing cardiac contractility without affecting heart rate [5]. AMG 986 was initially developed as a treatment for patients with $\mathrm{HF}$, and its chemical structure is presented in Fig. 1.

Recently, a phase $1 \mathrm{~b}$, first-in-human study in healthy and HF participants (NCT03276728) reported that AMG 986 exposure increased non-linearly with increasing oral doses of $5 \mathrm{mg}$ to $650 \mathrm{mg}$, with a trend of decreasing dosenormalized exposures observed across multiple ascending dose groups [4]. The oral bioavailability of AMG 986 ranged from 40 to $80 \%$, and the terminal half-life $\left(t_{1 / 2}\right)$ of 


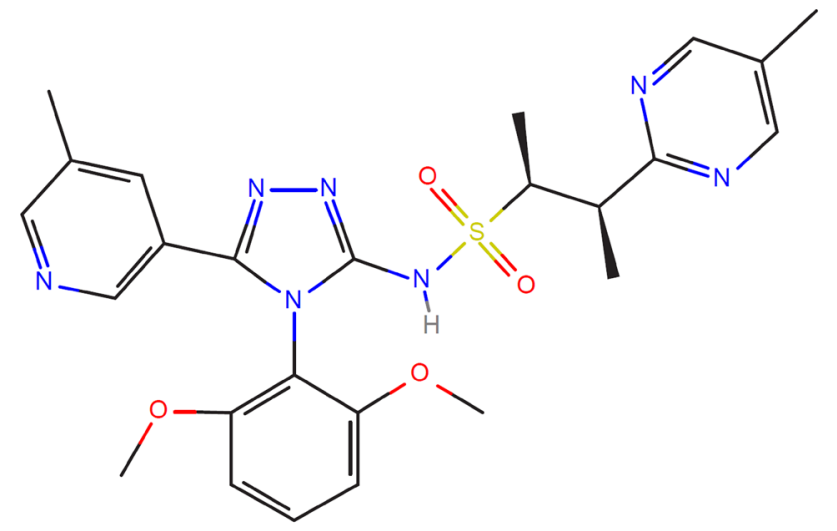

Fig. 1 Chemical structure of AMG 986.

AMG $986650 \mathrm{mg}$ was $21 \mathrm{~h}$; repeated once-daily dosing resulted in minimal accumulation across dose groups [4]. Additional studies indicate AMG 986 was highly protein bound (99.6\% bound in human plasma) and did not preferentially distribute into blood cells, and the in vitro unbound fraction in human plasma was 0.004. Furthermore, the metabolism of AMG 986 in vitro was principally catalyzed by human cytochrome P450 3A (CYP3A). AMG 986 was an in vitro inducer of CYP3A4, as determined by increases in CYP3A4 messenger RNA levels in primary human hepatocytes, and AMG 986 was not an in vitro inhibitor of any of the major drug-metabolizing human CYP enzymes. Additionally, AMG 986 was characterized in vitro as a substrate of P-glycoprotein and organic aniontransporting polypeptide 1B3 (Amgen, data on file).

The relationship between HF and renal impairment (RI) is well-established, with acute worsening of cardiac output leading to renal dysfunction (defined as an estimated glomerular filtration rate [eGFR] of $<90 \mathrm{~mL} / \mathrm{min} / 1.73 \mathrm{~m}^{2}$ ) [6, 7]. Further, many HF therapies result in acute renal failure or worsening of preexisting chronic kidney dysfunction [8, 9]. Broadly, RI can significantly alter intestinal, renal, and hepatic drug metabolism, increasing the risk of adverse events and resulting in the need for dose adjustments [10, 11]. Of note, RI may impact nonrenal clearance mechanisms through the downregulation of CYP enzymes [12].

Therefore, the objective of the current phase 1 study was to evaluate the safety, tolerability, and pharmacokinetics (PK) of a single oral 200-mg dose of AMG 986 in participants with severe RI compared with participants with normal renal function. Selection of the 200-mg dose of AMG 986 was based on data available when this study was planned, which demonstrated acceptable safety and tolerability of a single oral dose of up to $400 \mathrm{mg}$, and multiple daily oral doses of $200 \mathrm{mg}$.

\section{Methods}

\subsection{Study Design}

This was a phase 1, open-label, single-dose study conducted at four sites in the United States (US) (NCT03318809). The study consisted of a 28-day screening period followed by a 30-day treatment period. On day 1 , enrolled participants received a single oral dose of AMG $986200 \mathrm{mg}$ on an empty stomach (no food or liquids, except water, for at least $8 \mathrm{~h}$ before dose administration) and remained in a fasted state (no food or liquids, except water) for at least $2 \mathrm{~h}$ after dose administration. Participants resided at the research facility until day 2 for safety assessments and collection of blood samples for PK assessments, after which they were discharged and followed through to day 30; participants returned to the research facility at specified time points between days 2 and 22 for the completion of study assessments. An endof-study visit occurred on day 30 .

\subsection{Participants}

Eligible participants were males or nonchildbearing females (permanently sterile or postmenopausal) aged $18-65$ years with a body mass index of $18-38 \mathrm{~kg} / \mathrm{m}^{2}$. Laboratory test values were within normal limits for participants with normal renal function or consistent with the underlying condition for participants with severe RI, or clinically acceptable to the investigator for both normal renal function and participants with severe RI. Participants were required to be non-hypertensive or have treated, stable hypertension $(\leq 170 / 100 \mathrm{mmHg}$ ) during screening and day -1 ; for participants with severe RI, no change in dosage and medication was allowed for $\geq 4$ weeks prior to screening, and participants were expected to remain on a stable dose and medication for the entire duration of the study. Exclusion criteria included the concurrent or prior use of strong CYP3A4 inhibitors or strong CYP3A4 inducers within 14 days and 30 days, respectively, of day 1 .

Participants were assigned to one of two groups based on their eGFR, calculated with the Modification of Diet in Renal Disease equation [13]: normal renal function (eGFR $\geq 90 \mathrm{~mL} / \mathrm{min} / 1.73 \mathrm{~m}^{2}$ ) and severe RI (eGFR $15-29 \mathrm{~mL} /$ $\mathrm{min} / 1.73 \mathrm{~m}^{2}$ ). Participants in the severe RI group were not anticipated to require hemodialysis or renal transplantation and were anticipated to have renal function appropriate to severe RI for the duration of the study. Participants with normal renal function were matched to the severe RI group by age $( \pm 5$ years), body weight $( \pm 10 \%)$, proportion of men and women, and race. 


\subsection{Pharmacokinetic Sampling and Outcomes}

On day 1, blood samples for the PK analysis of AMG 986 were collected from each participant at pre-dose and 1, 2, 4, $6,8,12,24,48,72,96$, and 120 h post-dose (day 6) using $\mathrm{K}_{2}$ EDTA collection tubes. The PK parameters assessed were the maximum observed concentration $\left(C_{\max }\right)$, time to $C_{\max }$ $\left(t_{\max }\right)$, area under the curve (AUC) from time zero to the last quantifiable time point post-dose $\left(\mathrm{AUC}_{\text {last }}\right)$, AUC from time zero to infinity $\left(\mathrm{AUC}_{\mathrm{inf}}\right), t_{1 / 2}$, and apparent drug clearance observed after extravascular administration (CL/F). All PK parameters were estimated using noncompartmental analysis by Phoenix ${ }^{\circledR}$ WinNonlin ${ }^{\circledR}$ version 6.4 (Certara, Princeton, NJ, US).

Plasma concentrations of AMG 986 in human plasma were determined using validated high-performance liquid chromatography (HPLC) mass spectrometry. The analyte AMG 986 and internal standard (IS) ${ }^{13} \mathrm{C}_{6}$-AMG 986 were extracted from $0.050 \mathrm{~mL}$ of human plasma by a protein precipitation extraction procedure. The extraction began by adding $50.0 \mu \mathrm{L}$ of calibration standards, quality control samples, and study samples to appropriate wells of a 96-well plate, with $50.0 \mu \mathrm{L}$ of blank human plasma added to each blank well. The samples were diluted with $50 \mu \mathrm{L}$ of methanol/water $(50 / 50$, volume per volume $[v / v])$. Following the addition of $150 \mu \mathrm{L}$ of IS to all appropriate wells (or 150 $\mu \mathrm{L}$ of acetonitrile to blank wells), the plate was covered, vortexed, and centrifuged. A Tomtec Quadra4 ${ }^{\mathrm{TM}}$ (Tomtec Inc., CT, US) 96-well pipettor system was used to transfer $100 \mu \mathrm{L}$ of the supernatant to a new 96-well plate. After $400 \mu \mathrm{L}$ of acetonitrile/water (80/20, v/v) was added to all wells, the plate was covered and vortexed. The extracts were chromatographed under reverse-phase conditions on a Kinetex ${ }^{\circledR} C_{8}$ (Phenomenex Inc., CA, US) HPLC column using a gradient system with $0.1 \%$ formic acid in water and $0.1 \%$ formic acid in acetonitrile. The compounds were detected and quantified by tandem mass spectrometry in positive ion mode on an API $4000^{\mathrm{TM}}$ (AB Sciex, MA, US) equipped with a Turbo IonSpray ${ }^{\circledR}$ interface. The $\mathrm{m} / z$ transition values for AMG 986 and IS were $524.3 \rightarrow 312.2$ and $530.3 \rightarrow 318.2$, respectively. The assay had a lower limit of quantitation (LLOQ) of $10.0 \mathrm{ng} / \mathrm{mL}$. Calibration curves were obtained by performing a linear regression (weighted $1 / x^{2}$ ) on the calibration standards.

\subsection{Safety Outcomes}

The safety and tolerability of AMG 986 was assessed and included the subject incidence of treatment-emergent adverse events (TEAEs), serious adverse events (SAEs), clinical laboratory tests, 12-lead electrocardiogram (ECG), and vital signs. All adverse events were graded according to the Common Terminology Criteria for Adverse Events (CTCAE) version 4.0.

\subsection{Statistical Analysis}

Statistical analyses were performed using SAS version 9.3 (SAS Institute Inc., Cary, NC, US). No formal statistical hypothesis testing was performed. The sample size of this study was based on practical considerations and is consistent with the number of participants enrolled in similar studies. The sample size was confirmed to provide a $90 \%$ confidence interval (CI) of 0.81-1.23 for the geometric least squares mean (GLSM) ratio of the PK parameters. Concentrations below the LLOQ $(10.0 \mathrm{ng} / \mathrm{mL})$ were set to 0 before data analysis. GLSMs and 90\% CIs for the ratio of the GLSM (severe RI group/normal renal function group) were estimated using an analysis of variance model. The model used log-transformed PK parameters as the dependent variable and renal function group as the independent variable. The mean difference for the two groups was back-transformed to produce the GLSM ratio. Safety results were summarized using descriptive statistics.

\section{Results}

\subsection{Disposition of Participants and Baseline Characteristics}

Overall, 12 participants were enrolled in the study, and six participants each were allocated to the normal renal function and severe RI groups. All the 12 participants received a single 200-mg dose of AMG 986 and completed the study. The mean (standard deviation [SD]) age of participants in the severe RI and normal renal function groups was 56.7 (8.1) years and 57.8 (3.3) years. The mean (SD) weight of participants in the severe RI and normal renal function groups was 85.0 (13.0) $\mathrm{kg}$ and 82.1 (6.2) $\mathrm{kg}$ (Table 1).

\subsection{Effect of Severe RI on AMG 986 PK}

Mean plasma concentration-time profiles for AMG 986 $200 \mathrm{mg}$ were similar between normal renal function and severe RI groups (Fig. 2). Plasma PK parameters and statistical comparisons for AMG $986200 \mathrm{mg}$ between renal function groups are summarized in Table 2. After a single oral dose of $200 \mathrm{mg}$, plasma concentrations of AMG 986 peaked with a median $t_{\max }$ of $1.5 \mathrm{~h}$ in participants with normal renal function compared with $1.1 \mathrm{~h}$ in participants with severe RI. The geometric mean $t_{1 / 2}$ of AMG $986200 \mathrm{mg}$ was $21.1 \mathrm{~h}$ in participants with normal renal function versus 18.4 $\mathrm{h}$ in participants with severe RI. Geometric mean CL/F was 
Table 1 Baseline characteristics of participants

\begin{tabular}{lcc}
\hline Characteristic & Severe RI $(n=6)$ & $\begin{array}{l}\text { Normal renal } \\
\text { function }(n=6)\end{array}$ \\
\hline $\begin{array}{l}\text { Age, years, mean (SD) } \\
\text { Sex, } n(\%)\end{array}$ & $56.7(8.1)$ & $57.8(3.3)$ \\
Male & $3(50.0)$ & $3(50.0)$ \\
Female & $3(50.0)$ & $3(50.0)$ \\
Weight, kg, mean (SD) & $85.0(13.0)$ & $82.1(6.2)$ \\
Ethnicity, $n(\%)$ & & $1(16.7)$ \\
Hispanic/Latino & $0(0.0)$ & $5(83.3)$ \\
Not Hispanic/Latino & $6(100.0)$ & $2(33.3)$ \\
Race, $n(\%)$ & & $3(50.0)$ \\
White & $2(33.3)$ & $1(16.7)$ \\
Black or African American & $3(50.0)$ & \\
Asian & $1(16.7)$ & \\
\hline
\end{tabular}

$R I$ renal impairment, $S D$ standard deviation

$3040 \mathrm{~mL} / \mathrm{h}$ in participants with normal renal function and $2480 \mathrm{~mL} / \mathrm{h}$ in participants with severe RI (Table 2). Relative to exposures in participants with normal renal function, AMG $986200 \mathrm{mg} C_{\max }$ increased 1.41-fold (90\%
CI 0.88-2.27) and $\mathrm{AUC}_{\text {inf }}$ increased 1.23-fold (90\% CI 0.73-2.06) in participants with severe RI (Table 2).

\subsection{Safety}

A total of three participants (25.0\%) had TEAEs: two participants in the severe RI group (headache and dyspnea in one participant each) and one participant in the normal renal function group (nausea and dizziness). All TEAEs were CTCAE grade 1 in severity and not considered treatment related by the investigator. There were no SAEs, fatal adverse events, or adverse events leading to the discontinuation of treatment. No clinically important changes from baseline in laboratory values, ECG parameters, or vital signs were observed in either group.

\section{Discussion}

AMG 986 is a first-in-class, novel apelin receptor small molecule initially developed as a treatment for $\operatorname{HF}[4,5]$. In patients with HF, RI is a common comorbidity that can

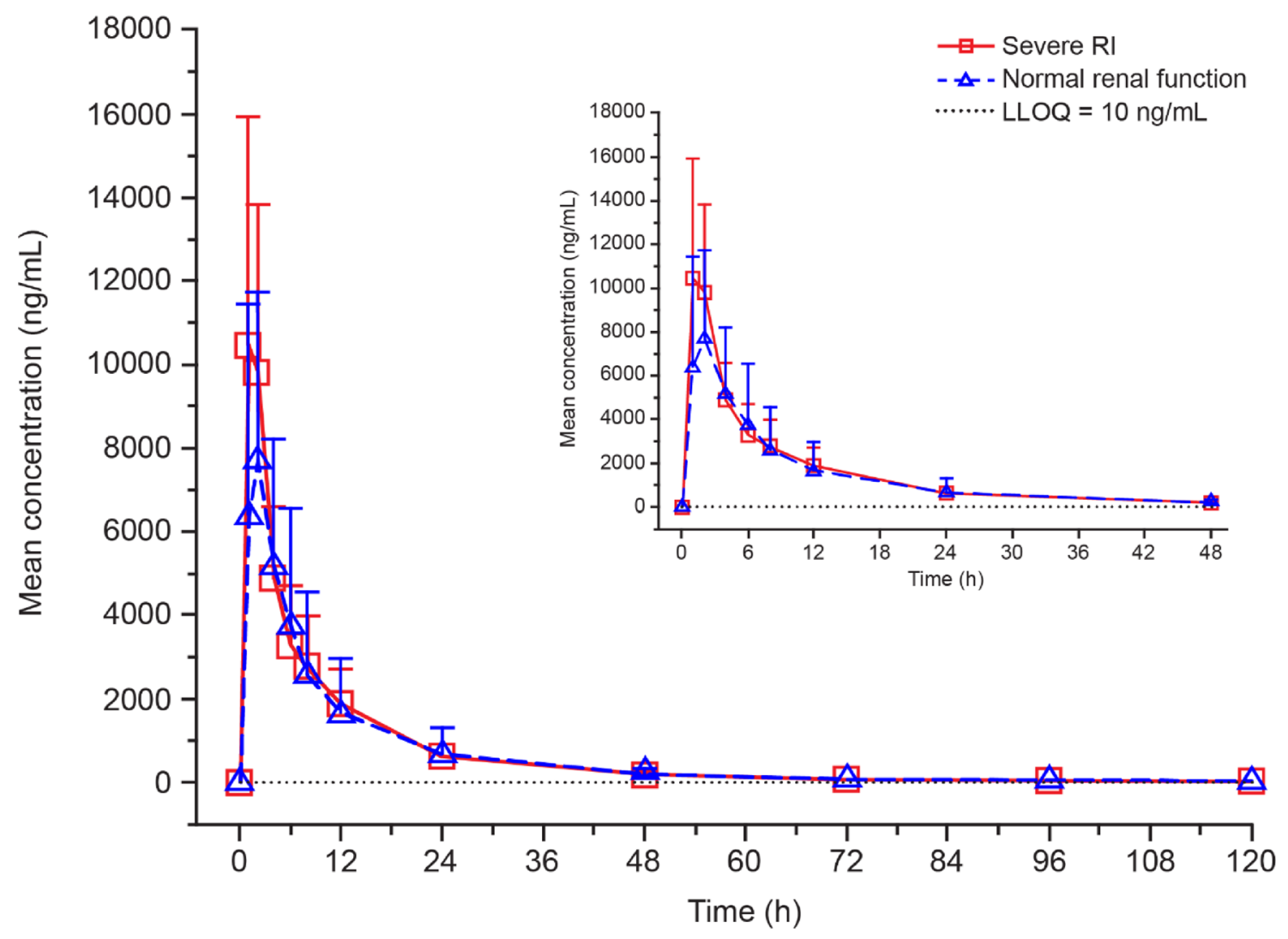

LLOQ, lower limit of quantitation; RI, renal impairment; SD, standard deviation.

Fig. 2 Mean (SD) plasma concentration-time profiles for AMG $986200 \mathrm{mg}$ administered orally to participants with severe RI and normal renal function (inset $=$ time 0 to 48 hours). $L L O Q$ lower limit of quantitation, $R I$ renal impairment, $S D$ standard deviation 
Table 2 Summary and statistical analyses of the effect of severe RI on AMG 986200 mg pharmacokinetic parameters

\begin{tabular}{|c|c|c|}
\hline Parameter & Severe RI (test) $(n=6)$ & $\begin{array}{l}\text { Normal renal func- } \\
\text { tion (reference) } \\
(n=6)\end{array}$ \\
\hline \multicolumn{3}{|l|}{$C_{\max }, \mathrm{ng} / \mathrm{mL}$} \\
\hline Geometric mean (geometric CV\%) & $10,600(45.3)$ & $7520(50.5)$ \\
\hline GLSM & $10,598.0$ & 7522.4 \\
\hline GLSM ratio test/reference $(90 \% \mathrm{CI})$ & $1.41(0.88-2.27)$ & - \\
\hline \multicolumn{3}{|l|}{$\mathrm{AUC}_{\text {last }}, \mathrm{h} \bullet \mathrm{ng} / \mathrm{mL}$} \\
\hline Geometric mean (geometric CV\%) & $80,000(33.6)$ & $64,500(70.0)$ \\
\hline GLSM & $80,011.8$ & $64,501.2$ \\
\hline GLSM ratio test/reference $(90 \% \mathrm{CI})$ & $1.24(0.73-2.10)$ & - \\
\hline \multicolumn{3}{|l|}{$\mathrm{AUC}_{\mathrm{inf}}, \mathrm{h} \bullet \mathrm{ng} / \mathrm{mL}$} \\
\hline Geometric mean (geometric CV\%) & $80,800(33.6)$ & $65,800(68.8)$ \\
\hline GLSM & $80,753.7$ & $65,842.9$ \\
\hline GLSM ratio test/reference $(90 \% \mathrm{CI})$ & $1.23(0.73-2.06)$ & - \\
\hline \multicolumn{3}{|l|}{$t_{\max }, \mathrm{h}$} \\
\hline Median (range) & $1.1(1.0-2.1)$ & $1.5(1.1-4.1)$ \\
\hline \multicolumn{3}{|l|}{$t_{1 / 2}, \mathrm{~h}$} \\
\hline Geometric mean (geometric CV\%) & $18.4(21.1)$ & $21.1(44.6)$ \\
\hline \multicolumn{3}{|l|}{$\mathrm{CL} / \mathrm{F}, \mathrm{mL} / \mathrm{h}$} \\
\hline Geometric mean (geometric CV\%) & $2480(33.6)$ & $3040(68.8)$ \\
\hline
\end{tabular}

$A U C_{i n f}$ area under the plasma concentration-time curve from time zero to infinity, $A U C_{\text {last }}$ area under the plasma concentration-time curve from time zero to the last quantifiable time point post-dose, $C I$ confidence interval, $C L / F$ apparent drug clearance observed after extravascular administration, $C_{\max }$ maximum observed plasma concentration, $C V \%$ coefficient of variation, GLSM geometric least squares mean, $R I$ renal impairment, $t_{1 / 2}$ terminal half-life, $t_{\max }$ time to maximum concentration influence the efficacy and safety of drug therapies [6-10]. This phase 1 study assessed the safety, tolerability, and PK of AMG $986200 \mathrm{mg}$ in participants with severe RI relative to participants with normal renal function. Per the US Food and Drug Administration (FDA) regulatory guidance for the assessment of drug PK in participants with impaired renal function, the current study used a "reduced PK study design" that included participants with severe RI only [11]. The inclusion of participants with severe RI, as opposed to a range of subgroups including participants with mild and moderate RI, represents the worst-case clinical scenario and allowed for the assessment of the greatest potential impact of impaired renal function on the PK of AMG 986 [11].

The results indicate that the observed half-life of AMG $986200 \mathrm{mg}$ was similar between participants with severe RI and normal renal function. The geometric mean CL/F was similar in participants with severe RI to that in participants with normal renal function, and AMG $986200 \mathrm{mg}$ exposures in participants with severe RI were not meaningfully different from exposures in participants with normal renal function, with a $\leq 24 \%$ increase in AMG $986200 \mathrm{mg}$ AUC in participants with severe RI. These results demonstrate that the PK of AMG $986200 \mathrm{mg}$ is minimally affected by renal function and can therefore be administered safely to patients with severe RI without the need for dose adjustments. In terms of safety, AMG 986 was generally well-tolerated; TEAEs were mild in severity, and there were no SAEs or fatal adverse events.

There are limitations to the current study. In the firstin-human study, AMG 986 was evaluated with single and multiple daily ascending doses up to $650 \mathrm{mg}$ [4]; however, the current study used a lower, single dose of $200 \mathrm{mg}$ because the limited data available when this study was planned indicated that this dose had acceptable safety and tolerability. Additionally, the plasma protein binding of AMG 986 was not evaluated in this study; thus, further studies are required to evaluate whether protein binding is altered in participants with RI. Per FDA regulatory guidance [11], a subsequent "full PK study design" using the identified therapeutic dose of AMG 986 and a regression analysis of PK parameter estimates in participants with varying degrees of renal function may be performed during the clinical development of AMG 986. Moreover, population PK analyses of data from subsequent phase 2 and/ or phase 3 clinical trials including patients with RI may be useful to characterize the impact of RI on AMG 986 exposure and provide appropriate dosing recommendations. 


\section{Conclusions}

In the current study, AMG $986200 \mathrm{mg}$ exposure, as measured by $C_{\max }$ and $\mathrm{AUC}_{\text {inf }}$, was similar in participants with severe RI and normal renal function. AMG 986 had an acceptable safety profile, with no SAEs or fatal adverse events. The results of this study support the enrollment of HF patients with RI into clinical trials of AMG 986 without the need for dose adjustments.

Acknowledgements Medical writing support, which was in accordance with Good Publication Practice (GPP3) guidelines, was provided by Liam Gillies, PhD, CMPP, of Cactus Life Sciences (part of Cactus Communications), funded by Amgen Inc.

\section{Declarations}

Funding This study was funded by Amgen Inc.

Conflict of interest/Competing interests AT, OM, SV, MAS, JH, and EL are employees and shareholders of Amgen Inc.

Availability of data and material Qualified researchers may request data from Amgen clinical studies; complete details are available at http:// www.amgen.com/datasharing.

Code availability Not applicable.

Ethics approval The study was conducted following ethical guidelines of the Declaration of Helsinki and Council for International Organizations of Medical Sciences, applicable Good Clinical Practice guidelines of the International Council for Harmonisation, and applicable local laws and regulations. Locally appointed ethics review boards (Aspire IRB [Santee, CA, US], Copernicus Group IRB [Cary, NC, US], and IntegReview IRB [Austin, TX, US]) approved the research protocol.

Consent to participate Written informed consent was obtained from all participants.

Consent for publication Not applicable.

Author contributions All authors provided substantial contributions to the conception or design of the study; or the acquisition, analysis, or interpretation of data for the study; and drafted the manuscript or revised it critically for important intellectual content; and gave final approval of the manuscript to be published.

Open Access This article is licensed under a Creative Commons Attribution-NonCommercial 4.0 International License, which permits any non-commercial use, sharing, adaptation, distribution and reproduction in any medium or format, as long as you give appropriate credit to the original author(s) and the source, provide a link to the Creative
Commons licence, and indicate if changes were made. The images or other third party material in this article are included in the article's Creative Commons licence, unless indicated otherwise in a credit line to the material. If material is not included in the article's Creative Commons licence and your intended use is not permitted by statutory regulation or exceeds the permitted use, you will need to obtain permission directly from the copyright holder. To view a copy of this licence, visit http://creativecommons.org/licenses/by-nc/4.0/.

\section{References}

1. Francis GS. Pathophysiology of chronic heart failure. Am J Med. 2001;110(Suppl 7A):37S-46S.

2. Jones NR, Roalfe AK, Adoki I, et al. Survival of patients with chronic heart failure in the community: a systematic review and meta-analysis. Eur J Heart Fail. 2019;21(11):1306-25.

3. Ma Z, Song J-J, Martin S, et al. The Elabela-APJ axis: a promising therapeutic target for heart failure. Heart Fail Rev. 2021;26;1249-1258.

4. Hellawell J, Abbasi S, Trivedi A, et al. Safety, tolerability, pharmacokinetics, and pharmacodynamics of AMG 986, a novel small molecule apelin receptor agonist, in healthy subjects and heart failure patients. J Cardiac Fail. 2020;26(10):S68.

5. Ason B, Chen Y, Guo Q, et al. Cardiovascular response to smallmolecule APJ activation. JCI Insight. 2020;5(8):e132898.

6. Damman K, Valente MA, Voors AA, et al. Renal impairment, worsening renal function, and outcome in patients with heart failure: an updated meta-analysis. Eur Heart J. 2014;35(7):455-69.

7. Schefold JC, Filippatos G, Hasenfuss G, et al. Heart failure and kidney dysfunction: epidemiology, mechanisms and management. Nat Rev Nephrol. 2016;12(10):610-23.

8. Schoolwerth AC, Sica DA, Ballermann BJ, et al. Renal considerations in angiotensin converting enzyme inhibitor therapy: a statement for healthcare professionals from the Council on the Kidney in Cardiovascular Disease and the Council for High Blood Pressure Research of the American Heart Association. Circulation. 2001;104(16): 1985-91.

9. Momoniat T, Ilyas D, Bhandari S. ACE inhibitors and ARBs: managing potassium and renal function. Cleve Clin J Med. 2019;86(9):601-7.

10. Dreisbach AW, Lertora JJ. The effect of chronic renal failure on drug metabolism and transport. Expert Opin Drug Metab Toxicol. 2008;4(8):1065-74.

11. US Food and Drug Administration. Pharmacokinetics in patients with impaired renal function-study design, data analysis, and impact on dosing and labeling. 2020. https://www.fda.gov/media/ 78573/download. Accessed Jun 2021.

12. Ladda MA, Goralski KB. The effects of CKD on cytochrome P450-mediated drug metabolism. Adv Chronic Kidney Dis. 2016;23(2):67-75.

13. Levey AS, Stevens LA, Schmid $\mathrm{CH}$, et al. A new equation to estimate glomerular filtration rate. Ann Intern Med. 2009;150(9):604-12. 